\title{
La evaluación de políticas y su institucionalización en España
}

Juan Antonio Garde Roca*

Palabras clave: Evaluación de políticas públicas, institucionalización de la evaluación, nueva gestión pública, gobernanza, agencias de evaluación, Agencia Estatal de Evaluación de las Políticas Públicas y la Calidad de los Servicios.

\section{Introducción}

La evaluación de políticas públicas desde vertientes y perspectivas diversas, ha sido tratada en las publicaciones $^{1}$ del Instituto Nacional de Administración Pública en distintas ocasiones y tiempos políticos, del mismo modo que ha sido objeto de estudio para los expertos españoles en múltiples foros y publicaciones, en una línea similar a las reflexiones y experiencias desarrolladas internacionalmente.

Precisamente, la importancia dada a la evaluación de políticas y a sus repercusiones para la Administración se refleja en los múltiples trabajos académicos y estudios elaborados tanto desde el ámbito gubernamental de distintos países como por los organismos especializados internacionales. Desde Administraciones como la de los EE.UU., pionera en la evaluación de políticas, programas, presupuestos y servicios federales, hasta las más próximas de nuestro entorno como las de Reino Unido, Francia o Suecia, se han desarrollado diversas experiencias de carácter muy heterogéneo y de notable interés, particularmente para la Unión Europea, que también viene incidiendo en la relevancia de la evaluación de sus políticas y en la necesidad de su desarrollo por parte de sus países miembros.

Del mismo modo, y de manera creciente, la importancia se denota en las experiencias de muchas organizaciones multilaterales dedicadas a la cooperación y ayuda al desarrollo, como Naciones Unidas, el Banco Mundial o el Banco Interamericano de Desarrollo, que estimulan y fomentan las evaluaciones de programas por parte de las Administraciones públicas de los países receptores de las ayudas, bien a partir de la publicación de nume-

* Presidente de la Agencia Estatal de Evaluación de las Políticas Públicas y la Calidad de los Servicios.

${ }^{1}$ Véanse, por ejemplo, los números 3 (mayo-agosto de 1995) y 11-12 (enero-agosto de 1998) de la revista Gestión y Análisis de Políticas Públicas, así como los números 224 y 225 (octubre de 1990-marzo de 2001) de Documentación Administrativa. 
rosos estudios teóricos , bien por medio de guías o manuales prácticos de notoria consideración. Similar interés por la evaluación se evidencia en los informes, recomendaciones y manuales de la OCDE, desde aquellos realizados en los años 80 con sus propuestas de reformas de las Administraciones públicas y concretamente en el servicio al público ${ }^{2}$, hasta los más recientes de este nuevo siglo, como fue la recomendación referida en 2005 al proceso español de creación de la Agencia Estatal de Evaluación ${ }^{3}$, por citar un ejemplo ahora pertinente. Todos ellos son someros referentes internacionales necesarios para contextualizar el momento presente de las Administraciones respecto de la evaluación de las políticas y la calidad de los servicios públicos.

Por ello valoro muy especialmente que la revista Gestión y Análisis de Políticas Públicas dedique nuevamente un número monográfico a las experiencias y avances en este ámbito, así como a profundizar en los argumentos y las finalidades últimas de una mayor institucionalización de la evaluación en nuestro país.

La necesidad de la evaluación de las políticas y programas públicos goza de una rara unanimidad, tanto dentro del ámbito académico como del político, y viene respaldada por más de ocho décadas de práctica y por una muy abundante literatura económica, social y política al respecto. Pero esta falta de contestación a su necesidad contrasta profundamente con las graves limitaciones de sus usos, lo cual viene motivado por los problemas de diseño y ejecución de las propias políticas y programas públicos, por las contradicciones de objetivos de los mismos, por las dificultades para la medición de resultados e impactos, por las reticencias de los poderes públicos y los propios gestores a su utilización y publicidad efectiva, así como por las limitaciones y contenidos de la propia institución, incapaz de responder, en ocasiones por su obsolescencia, a las nuevas necesidades de la gestión pública y la gobernanza. A pesar de todo ello, la evaluación es, sin duda, uno de los elementos más claves de la nueva gobernanza, capaz de ofrecer una respuesta positiva a la necesidad de racionalizar el uso de los recursos públicos y mejorar la calidad del gasto y de responder simultáneamente a los retos de ciudadanía y de calidad democrática de nuestras sociedades.

La evaluación, tal como ha sido definida por el Consejo Científico de Evaluación francés, es una actividad «institucional que tiene vocación de integrarse en la gestión pública y en el funcionamiento del sistema político», y que solo alcanza su sentido y perspectiva más profunda si se inserta como una fase del desarrollo de las políticas públicas, diseñada y puesta en práctica bajo la supervisión y responsabilidad de una autoridad pública, para atender a sus objetivos específicos de aprendizaje y mejora del servicio de los ciudadanos.

Entendemos por «institucionalización de la evaluación» el proceso político por el que se legitima un marco normativo adecuado para el desarrollo de la función de la evaluación, a partir de la estructuración de espacios e instancias de las Administraciones a las que encargar tal función, ubicándolos en los distintos niveles de gobierno y según las distintas actividades o sectores a evaluar.

Con la institucionalización no se trata sólo de realizar algunas evaluaciones, sino también de incorporar la evaluación y sus resultados como prioridad de la agenda gubernamental dentro de los procesos de formación de políticas públicas, haciendo de ellas un factor clave de gobernanza que ayude a arbitrar la participación de la ciudadanía y de los distintos actores sociales.

${ }^{2}$ El informe de la OCDE de 1986, aparecido en 1987 como documento publicado con el título de La Administración al servicio del público, es un clásico en las líneas de trabajo de modernización y mejoras prácticas en los países desarrollados. Fue publicado con el mismo título por el Ministerio de Administraciones Públicas en 1988.

${ }^{3}$ En Economic Survey. Spain 2005 (OCDE 2005) se hace referencia a la creación de la Agencia de Evaluación como parte del compromiso del Gobierno español con la mejora de la calidad de la información sobre los servicios públicos, señalando que, para ser efectiva, la Agencia deberá contar con suficientes recursos e independencia. La versión española de dicho Informe puede encontrarse en la web 〈http://www.oecd.org/dataoecd/53/54/34599244.pdf). 
Institucionalizar la evaluación exige configurar un modelo permanente de análisis de resultados, definiendo los principios y valores en los que sustentar dichos análisis y evaluaciones y enmarcándolos dentro de un proyecto político de gobernanza. Para ello, los poderes públicos, respetando íntegramente los roles legítimos del poder decisorio, deben estimular el establecimiento de estructuras culturales y formales capaces de diseñar sistémicamente e implementar las políticas públicas, así como de definir recursos técnicos, normas, metodologías, etc. que hagan de la evaluación de tales políticas un proceso riguroso, transparente y de calidad, al servicio de los ciudadanos. Serán éstos, junto con los diversos actores sociales a través de su participación, los que expresen sus demandas, necesidades y opiniones, y a los que, en todo caso, se les debe rendir cuenta de los resultados de las evaluaciones realizadas.

A lo largo de este artículo, situaremos a la evaluación en su contexto histórico, a través del devenir del propio concepto y de sus prácticas hasta el momento presente, en el que la enmarcaremos desde la perspectiva de la gobernanza. Ello conllevará la necesaria referencia a la multiplicidad de actores interesados en la evaluación y el nuevo contexto de la sociedad del conocimiento, así como la asunción gubernamental del compromiso de institucionalización de un sistema público de evaluación, traducido en el mandato concreto de creación de la Agencia Estatal de Evaluación de las Políticas Públicas y la Calidad de los Servicios, sobre la que, finalizando el artículo, se enunciarán algunos elementos que, a mi juicio, deben caracterizar su contenido y funcionamiento.

\section{Perspectivas de la evaluación ${ }^{4}$}

Por citar algunos elementos que la caracterizan, se puede definir a la evaluación como el proceso sistemático de recolección y análisis de la información, destinado a describir la realidad y emitir juicios de valor sobre su adecuación a un patrón o criterio de referencia establecido como base para la toma de decisiones. Evaluar es participar en la construcción de un tipo de conocimiento axiológico, interpretando la información obtenida, estableciendo visiones no simplificadas de la realidad y suministrando resultados basados en «evidencias que sean creíbles, fiables y útiles, facilitando la incorporación oportuna de los hallazgos, recomendaciones y lecciones en los procesos de toma de decisiones» (Naciones Unidas 2005), lo cual ayudará a generar una mayor cultura evaluativa5.

De forma más pragmática, otros autores estiman que evaluar es elaborar «unas pautas de referencia que conjuguen criterios operativos y políticamente legítimos de la eficacia y los resultados de una política, formulando análisis vinculados a esas pautas que resulten pertinentes desde el punto de vista de la acción y la decisión, respondiendo de manera pragmática con el conjunto de herramientas de las ciencias sociales y de la gestión» (Perret 2001). La práctica de la evaluación incluye actividades técnicas de observación, medida y análisis, pero no se reduce sólo a eso. No es una disciplina científica en el sentido habitual del término, sino una «actividad institucional» (Perret 2001). Sus fronteras son fluidas con otras técnicas como el control, el análisis de gestión, el «benchmarking», la consultoría, la investigación aplicada o la auditoría, pero su finalidad está indisolublemente unida a los resultados e impactos de las políticas y programas en relación con los fines últimos pretendidos.

\footnotetext{
${ }^{4}$ Algunas de las reflexiones contenidas en este primer apartado de «perspectivas» y en el tercer apartado de «sociedad del conocimiento» están elaboradas a partir de las notas tomadas en una sesión de debate e intercambio de experiencias mantenida en el INAP, el 31 de mayo de 2005, con los expertos de evaluación Ray C. Rist, Nicoletta Stame y Osvaldo Néstor Feinstein.

${ }^{5}$ Entre las numerosas definiciones de evaluación asumidas internacionalmente, es conocida la realizada en 1991 en el seno de la OCDE/CAD por el «Grupo de expertos en Evaluación de Programas de Asistencia Internacional de la Organización de Cooperación y Desarrollo Económico/Comité de Asistencia para el Desarrollo», que define la evaluación como «un escrutinio -lo más sistemático y objetivo posible- de un proyecto, programa o política en ejecución o terminado, y sus dimensiones de diseño, ejecución y resultados. El propósito es determinar la pertinencia y logro de los objetivos y la eficiencia, efectividad, impacto y sustentabilidad del desarrollo».
} 
Más allá de las consideradas primeras evaluaciones de servicios públicos en los albores del siglo XX y que revistieron en los EE.UU. la forma de investigaciones sobre educación e infraestructuras hasta los años 30, puede afirmarse que la primera «oleada» histórica más consistente de estudios de políticas públicas se produjo en ese país en los primeros años 60 (Monnier 1995; Subirats 1989; Ferrera 1991), entendiendo la evaluación en esta etapa como un sinónimo de medición y cuantificación de resultados de la gestión pública al servicio de los gestores.

Ya en una segunda etapa histórica, a finales de los años 70, la evaluación deviene fundamentalmente en una descriptiva de los programas públicos puestos en marcha para la resolución de los problemas sociales en tanto que instrumento político para la reconsideración de políticas y presupuestos. El enfoque dominante que se propugnaba de la evaluación hasta ese momento era de carácter interno a la propia Administración y a los gobiernos.

Sin embargo, con la tercera oleada de los años 80 , la evaluación pasa a interesar también a los parlamentos (Derlien 1998), pasando a asimilarse al juicio sobre las políticas y programas y centrada especialmente en el estudio de los objetivos de los mismos y en el impacto social del cambio producido tras la intervención pública.

Seguramente las peculiaridades sociales y económicas de los EE.UU., presentes en las numerosas y heterogéneas metodologías de evaluación, ocasionó en su momento, como señalan algunos autores ${ }^{6}$, que los evaluadores debatieran profusamente en las publicaciones académicas de los EE.UU. sobre la naturaleza y origen de los estándares, la naturaleza de la causalidad y su relación con la validez, su utilidad y usos reales de los resultados de la evaluación, la participación activa de la población objetivo en la evaluación, la integración de intereses contrapuestos o la confiabilidad y objetividad de las diversas metodologías, entre otros aspectos de gran interés para el devenir de la evaluación.

Desde finales de los 80 , tras el «gran desarrollo que los estudios de evaluación de programas tuvieron en los EE.UU., se tiende a considerar a la evaluación como el método de investigación sistemática sobre la configuración de un programa o política determinada, y sobre su implementación y efectividad», admitiéndose por lo general «que los métodos de evaluación de los programas de actuación gubernamentales sirven tanto para un mejor conocimiento de los mismos por parte de la opinión pública como por parte de los protagonistas mismos de esos programas en la Administración Pública, que quieren beneficiarse de la información que genera la actividad evaluadora» (Subirats 1989).

La evaluación de las políticas no ha caminado siempre unida a la extensión de las responsabilidades de los poderes públicos (dirigiendo los asuntos sociales), sino que, a menudo, los Estados requerían el auxilio de la evaluación sólo cuando su legitimidad, relacionada con sus actuaciones, atravesaba momentos de debilidad, tal y como sucedió en EE.UU. en los años 50, por ejemplo, con las sucesivas crisis de legitimidad del modelo burocrático (Monnier 1995).

En los países europeos comenzó a extenderse inicialmente la práctica de la evaluación de políticas públicas a partir de la conceptualización y del aprendizaje de las experiencias que provenían de los EE.UU. Es a finales de los años 60 y coincidiendo con algunas crisis de legitimidad del Estado cuando «se produce una búsqueda activa de legitimación del lado de la ciencia» (Monnier 1995). Coincidiendo, con la primera oleada de estudios antes citada, en Europa comenzó a asumirse también la necesidad de incorporar la evaluación a los programas nacionales de auditoría y de control interno de las organizaciones del sector público.

${ }^{6}$ Entre otros, Rivera y Fernández y Zavala (2002). 
A partir de la profundización en la administración de los Fondos Estructurales de la Comunidad Europea ${ }^{7}$ para el desarrollo y equilibrio de sus Estados miembros, y de modo paralelo a lo sucedido con la tercera oleada de los estudios internacionales de finales de los años 80, los sistemas de evaluación se extienden y abarcan al conjunto de la sociedad civil, organizaciones no gubernamentales, etc., acometiéndose la evaluación desde las más diversas perspectivas académicas y profesionales e institucionalizándose en mayor medida la evaluación, fundamentalmente en los países del norte de Europa.

Con la cuarta oleada de estudios internacionales, en el entorno de los años 90, se sitúa a la evaluación dentro de un proceso de debate orientado por los valores e intereses de los distintos actores sociales. Es en este contexto de desarrollo histórico de la evaluación cuando en la mayoría de los países de la Unión Europea se producen movimientos internos favorables a la constitución de institutos o sociedades de evaluación, desarrollándose desde hace algo más de una década un amplio movimiento de personas e instituciones que se aglutinan a través de redes personales en las que se intercambian saberes y experiencias y que se articulan con otras redes internacionales, fundamentalmente situadas en América.

En la evolución metodológica, con las ya citadas oleadas de los estudios de evaluación, se vinieron acometiendo las valoraciones y juicios sobre los servicios, los programas, los proyectos, los planes, las políticas, etc., los cuales se apoyaron en numerosas y variadas técnicas, desde los análisis de coste/beneficio hasta las metodologías cuantitativas y cualitativas o la mezcla de todas ellas.

Con las metodologías desarrolladas los gobiernos han venido procurando dotarse de herramientas para conocer en cada momento o sobre cada problema, qué funciona mejor y qué peor de las políticas que se deciden democráticamente. Al mismo tiempo, con el aprendizaje sobre la calidad de las políticas públicas por parte de decisores, gobernantes e instituciones, se ha extendido la necesidad de rendir cuentas a la ciudadanía con mayor rigor y transparencia, a fin de legitimar socialmente la acción pública.

Lo cierto es que, por lo general, los resultados devenidos con el desarrollo de las distintas fases evolutivas de la evaluación, tanto en términos de la aplicación o del uso de la evaluación como de la rendición de cuentas a la sociedad, no han llegado a ser satisfactorios en muchos países en la medida en que los mecanismos existentes para la realización de las evaluaciones han venido siendo incompletos e insuficientes. Es por ello por lo que en España, como en otros países del mundo, la evaluación de políticas y la calidad de los servicios sigue ocupando hoy un lugar destacado en la agenda gubernamental de las reformas del sector público.

Construir una cultura de evaluación implica incorporar la evaluación como una práctica institucional cotidiana. La evaluación requiere de un proceso reflexivo y riguroso de análisis crítico para indagar la realidad, que permita emitir juicios y que sirva de apoyo para la toma de decisiones, razón por la cual el proceso debe regirse por principios de validez, participación y ética.

Sin embargo, a pesar del desarrollo metodológico producido, aún hoy no es posible verificar una relación causa-efecto entre las lecciones aprendidas a partir de las evaluaciones realizadas y la posterior formulación o mejora de las nuevas políticas o de los procesos en los que se sostiene la implementación de las mismas. De nuevo,

${ }^{7}$ Para ver la evolución y el marco reglamentario de los sistemas de evaluación y aplicación de técnicas cualitativas en la evaluación de los programas de desarrollo regional de la Unión Europea, puede consultarse Viñas (2001 y 2002). 
como en los EE.UU., en Europa se viene produciendo, por un lado, un crecimiento de la conciencia teórica respecto de la necesidad de aplicar lo aprendido con la evaluación y, por otro lado, una postergación de la puesta en práctica de estas lecciones que, en definitiva, supone el desaprovechamiento coyuntural de las mismas por parte de muchos decisores.

El uso instrumental de las evaluaciones, si fuese una práctica habitual de los poderes públicos, significaría un aprovechamiento continuo de los resultados de las mismas y una ampliación sobrevenida de conocimientos para aquellos poderes. En el supuesto contrario, la no utilización por parte de los decisores estaría significando una ineficiencia poco justificable en el marco de la rendición de cuentas al Parlamento y a la sociedad que toda esfera gubernamental debe realizar.

Por esta última razón, con la mayor institucionalización de la evaluación de las intervenciones públicas se promueve, de modo prioritario, la credibilidad y progresiva utilización de los resultados de las evaluaciones. De no hacerse así, se resquebraja la confianza en la finalidad de las mismas, no contribuyéndose con esa inhibición a la extensión de los valores plausibles de la cultura de evaluación que es asunto de gran calado para la gobernanza, como señalé en la introducción, en orden a la consecución de una mayor legitimidad social de la acción pública.

La cultura y, por supuesto, las prácticas de medición de los resultados e impactos por las organizaciones y decisores públicos, exigen la consideración de la evaluación como un instrumento que trasciende del control de los recursos públicos gestionados, que sirva, aunque no únicamente, para responder si lo que se ha realizado se ha hecho o no según lo previsto en el programa de intervención pública que lo sustenta. La evaluación, además, debe permitir preguntarse por el modo en que se produjo el diseño, la implementación y el despliegue de la política, así como por sus impactos sociales, facilitándose el mejor aprovechamiento de los resultados del proceso de evaluación para la mejora de la gobernanza. Se trata de un conjunto de prácticas que, tal como se viene señalando, implican la recopilación y el tratamiento de la información relevante sobre la acción pública (¿qué ha pasado?), el despliegue de juicios normativos sobre lo acaecido (¿está bien hecho?) y las preocupaciones instrumentales para su efectiva mejora (¿cómo hacerlo mejor?) ${ }^{8}$.

La evaluación, por tanto, es un factor clave para realimentar las distintas fases de la gestión pública, tanto las encaminadas a la implementación de las políticas, desde sus resultados, como las vinculadas a los procesos de planificación o formulación del presupuesto. Sin embargo, a pesar de ser una pieza esencial para la toma de decisiones de carácter presupuestario y el conocimiento de los resultados e impactos de los programas, la evaluación sigue siendo una asignatura pendiente en este ámbito y en buena parte de los países. Todavía hoy puede afirmarse que en la práctica institucional no se ha encontrado el diseño óptimo que permita incorporar a las decisiones presupuestarias la evaluación de resultados de las políticas, lo que probablemente deberá transitar mediante la incorporación al análisis de agencias de evaluación externas a las autoridades presupuestarias y a los departamentos interesados en la negociación (Zapico 2005).

La evaluación está llamada a ser, en consecuencia, una herramienta utilizada por todos los niveles de gobierno y un arma potente de vinculación de la teoría a las prácticas gubernamentales y administrativas, convirtiéndose en la mejor oportunidad para el desarrollo del buen gobierno.

${ }^{8}$ La definición de estas prácticas están señaladas en la Petit Guide de l'Evaluation des politiques publique, del Conseil Scientifique de l'Evaluation (1996). 


\section{La nueva gestión pública, gobernanza y evaluación}

Es aceptado que en las Administraciones públicas de los países más avanzados se vienen compartiendo similares líneas de reformas en materia de gestión pública. La gestión orientada a resultados, desarrollando indicadores que permitan medir eficacia, eficiencia y calidad, así como el fortalecimiento de los mecanismos de seguimiento y control del cumplimiento de objetivos son, junto a la herramienta de evaluación que debe impregnar a todas las fases de la gestión e implementación de políticas, algunos de los rasgos básicos que, entre otros, conforman la llamada «nueva gestión pública».

Como es conocido, con este concepto, aun no siendo un paradigma doctrinal de corpus homogéneo, se sintetizan las pautas dominantes de las múltiples experiencias internacionales de implantación de sistemas gerencialistas en las Administraciones públicas, especialmente en el mundo anglosajón.

Nuestro país, como el resto de países de la OCDE, necesita enmarcar sus procesos de reformas justamente dentro del contexto de estas nuevas claves de gerencia pública, para enfrentarse a los retos de una sociedad crecientemente compleja en un mundo cada vez más globalizado e interrelacionado, no sólo en el terreno económico, sino también en las relaciones políticas internacionales. Tales retos suponen para los gobiernos que dirigen la Administración pública definir prioridades como las siguientes:

- Cómo abordar mejor desde la esfera pública la intervención del Estado en la sociedad y, por tanto, cómo maximizar el interés público de la relación Estado-mercado, en un contexto de fuerte aumento de la competencia económica;

- Cómo abordar las demandas sociales de mayor calidad de vida, lo que supone asumir por las instituciones públicas la mejora y extensión de los contenidos y la calidad de los servicios públicos, adaptándolos a los retos demográficos y de esperanza de vida;

- Cómo impulsar una mayor calidad democrática y mejores pautas de gobernabilidad, con participación de múltiples actores, instituciones, sectores y niveles de gobierno, en el nivel nacional e internacional, estatal y territorial, con una creciente demanda de participación y control ciudadano sobre las acciones de gobierno y respecto de la administración de los dineros públicos.

Aunque es imprescindible formular políticas que den la debida respuesta a tales prioridades, la toma de decisiones no sólo debe dirigirse al desarrollo económico y social, al avance de los procesos de descentralización política y administrativa, a la mejora de la calidad de los servicios públicos y a los procesos de control de los mismos, sino que debe prestarse, al mismo tiempo, una atención especial a los mecanismos de concertación con los actores sociales, participación ciudadana y rendición de cuentas.

Desde una visión de planificación estratégica, las instituciones públicas deben dar respuestas a la incertidumbre de los cambios del entorno, a la complejidad de los procesos económicos, políticos y sociales, a la interdependencia institucional y a la heterogeneidad de los actores participantes que, lógicamente, demandan intereses distintos que deben ser gestionados democráticamente y desde el servicio a los intereses generales.

Dichas respuestas institucionales pueden darse no sólo modernizando el funcionamiento de las Administraciones públicas a partir del conocimiento de las mejores prácticas y experiencias, sino con la realización de los estudios prospectivos adecuados y el análisis de las políticas públicas y la evaluación de éstas y de sus impactos, de modo que se fortalezca la inserción supranacional del Estado, se robustezca la sintonía de éste con la ciudadanía y se atine en la satisfacción de las demandas sociales que legítimamente se invoquen. 
Efectivamente, para la adopción de determinadas decisiones públicas en la mayoría de los países desarrollados, en el contexto de cambios acelerados y baja previsibilidad, es un aspecto crucial el conocimiento de las experiencias e innovaciones que acontecen en el mundo respecto de los procesos reformadores del sector público, dado además el avance tecnológico por el que el acceso a la información y al conocimiento se realiza en tiempo real.

La modernización de las Administraciones, su organización y funcionamiento, el modo de dirigirlas y las técnicas de gestión a emplear se constituyen, como denominador común, en determinados componentes que conforman la nueva gerencia pública. La evaluación es uno de esos elementos, fundamental y crítico de las reformas, en la medida en que con ella también puede rendirse cuentas desde el Estado de manera más eficaz.

La insuficiente explicación y conocimiento de los resultados de las políticas públicas genera desconfianza en la ciudadanía y suscita críticas al funcionamiento y a la transparencia y a la calidad de algunos servicios públicos, lo que no contribuye precisamente a la legitimación social del funcionamiento del Estado.

En el contexto de integración europea, es creciente el requerimiento de mayor rigor, no sólo presupuestario, de la formulación de políicas por los distintos gobiernos, de unos y otros niveles, lo que incidiría también en las políticas estatales y su ámbito interno. Por ello, la gestión pública debe hacer efectiva la responsabilidad a través de renovados mecanismos de control de los recursos e incentivos públicos, con un mayor conocimiento del impacto, previsible o posterior, de las políticas públicas que se adoptan.

La supranacionalidad en la adopción de muchas decisiones políticas y el funcionamiento del Estado a través de la descentralización competencial en las Administraciones autonómicas crean nuevos procesos de toma de decisiones y nuevos cauces de comunicación entre los diversos niveles de gobierno y la sociedad que se ve segmentada en la recepción de muchas prestaciones públicas.

Pero para que esa comunicación de la ciudadanía con el Estado sea fructífera y con ella se renueven algunos elementos necesarios para la calidad democrática hay que disponer de estudios científicos y con información rigurosa a partir de la evaluación de las políticas que se pretendan formular o se estén desarrollando.

Esa información analítica sobre el contenido del impacto de las decisiones públicas es la que debe fluir por renovados canales de comunicación con la ciudadanía, con transparencia, para mejorar el aprendizaje en la toma de decisiones. La transparencia exige un esfuerzo por definir previamente objetivos y estrategias, así como los resultados que se esperan conseguir en la implementación de las políticas públicas, de lo que particularmente hay que dar cuenta a los actores involucrados y a los destinatarios y usuarios de los servicios públicos.

Con el diseño de nuevas líneas de gestión pública que incorporen las herramientas de evaluación, probablemente podrán formularse mejor las estrategias gubernamentales para la implementación de las políticas, las cuales serían mejor visualizadas y más comprendidas por la ciudadanía y los grupos de interés. Para ello, las instituciones deben incorporar a sus procesos de gestión nuevos sistemas de «evaluación intermedia» que faciliten un mejor seguimiento y control, lo cual incluso podría suponer la modificación o reconsideración del contenido de aquellas políticas así como de sistemas de «evaluación final» de las mismas con los que contrastar su validez, eficacia o pertinencia, e implicaría en la práctica una mayor explicitación de los compromisos entre decisores públicos y la sociedad.

Esos compromisos podrían enunciarse, además, a través del establecimiento de planes plurianuales de evaluaciones que, entre otras finalidades, dotasen a los poderes públicos de grandes «almacenes de conocimiento» 
que pudieran compartirse institucionalmente, además de convertirse en instrumentos necesarios con los que evaluar las mejoras reales y los avances de las prácticas evaluativas y de su progresivo crecimiento cuantitativo en el conjunto de las distintas Administraciones.

\section{Sociedad del conocimiento y multiplicidad de actores interesados en la evaluación}

Para que las instituciones, decisores y organizaciones públicas apliquen con éxito los resultados de las evaluaciones, no basta con disponer de unos informes aparentemente buenos y voluminosos si en su realización no se ha tenido en cuenta lo que algunos expertos denominan «corrientes de conocimiento» a las que ahora me referiré.

El desarrollo de la sociedad del conocimiento ha traído consigo una rápida acumulación de información pero, en general, no se ha producido todavía una eficaz gestión del conocimiento acumulado a fin de que las organizaciones gubernamentales no asuman meras actitudes reactivas o se vean desbordadas por la sucesión de acontecimientos sobre los que no tienen previsto la mejor manera de afrontarlos.

La presión ciudadana sobre los gobiernos ha venido creciendo en los últimos años; se les pide cada vez más eficacia y mayor y más pronta reactividad ante los problemas complejos que van presentándose en el mundo globalizado.

Hoy es posible acumular mucha información sobre la gestión de problemas similares en cualquier parte del mundo, pero a la vez esa acumulación informativa exige de analistas que, gestionando el conocimiento, faciliten la adopción de decisiones con el máximo de valoraciones que sea posible realizar, interaccionando de modo sinérgico unas y otras informaciones de unas y otras latitudes e instituciones gubernamentales del planeta. Ahí reside la esencia de esas «corrientes de conocimiento» que antes citaba.

Asimismo, también es viable el análisis sincrónico de multitud de informaciones, de cientos de conocimientos acumulados en potentes bases de datos en orden a la mejor gestión de las políticas públicas, escuchando las voces de los afectados, incluso a tiempo real, y no demorando burocráticamente los estudios a fechas ulteriores, sino produciendo juicios que den respuesta a las demandas sociales en un tiempo relativamente corto. Por ello, no resulta deseable que se desaprovechen las lecciones de las evaluaciones remitiendo su utilización a futuras fechas que, habitualmente y en su caso, nunca llegan a suponer un mejor contexto para ser aplicadas.

Se están produciendo también nuevas «corrientes de conocimiento» en el marco de los diversos discursos internacionales que generan un pensamiento evolucionado sobre la evaluación de políticas y que ponen en cuestión los usos y las prácticas que hasta ahora venían produciéndose en muchos lugares del mundo.

Antes, los informes de evaluación solían tener un único destinatario; ahora los usuarios de la evaluación son múltiples, pues se reconoce que múltiples son los stakebolders de la evaluación. Ésta no sólo se realiza para un gestor público que la encarga, de un país y una institución concreta, sino para ser almacenada también en generosas «bases de datos» que puedan ser compartidas por muchos otros usuarios de la misma, por muy alejados que estuvieran de aquel país e institución donde fue demandada la evaluación.

El modo actual del pensar colectivo, dada la complejidad de la sociedad, incluyendo a los gestores públicos y a los analistas y evaluadores de políticas, se produce en un marco de generación mucho más rápida de estudios y 
decisiones, más omnicomprensiva y más multidisciplinar que antaño. Se integran los métodos múltiples de evaluación (cuantitativa, cualitativa...), porque los problemas a resolver se han hecho más complejos y requieren de mayores perspectivas y solo una aproximación sistémica a los mismos puede ofrecer los resultados deseables.

En la sociedad del conocimiento, y dadas las características de complejidad que afectan a problemas, actores, políticas y soluciones a adoptar, la evaluación puede ofrecer alto valor añadido estratégico a la gestión pública, en tanto que permitiría la interrelación de los múltiples elementos conectados entre sí, aunque aparentemente no lo estuvieran, y cuya conexión, a través de la malla en la que se desarrollan las políticas públicas, puede constituirse en la explicación justificativa de sus resultados e impactos.

El contenido de los análisis y evaluación de políticas, en el contexto de la actual sociedad y dadas las potencialidades e interdependencias del conocimiento existente, no puede dejar de considerarse, ni demorarse o diferirse en el tiempo su toma de razón por los decisores, sin riesgo de adoptar soluciones alejadas de las complejas realidades en las que se desenvuelven hoy las políticas públicas, que, a su vez, son observadas y juzgadas por muchos otros actores sociales y grupos de intereses distintos a los decisores públicos.

Por esa razón, muchas organizaciones públicas, procurando la adopción de las mejores decisiones, valoran cada vez más la obtención de la máxima «información y conocimiento acumulado» y del modo más rápido posible, para lo que se asocian crecientemente en «alianzas» con su entorno («partenariados»), integrando, y no desaprovechando, la producción de conocimientos de, por ejemplo, universidades, hospitales, instituciones de investigación, centros de conocimiento, escuelas de negocios, sociedades de evaluación, empresas multinacionales, etc,, tanto del sector público como del privado, apoyándose para ello en el uso de la red poderosa que es Internet, y estimulando la creación de nuevos marcos de intercambio y transferencia de conocimientos para la evaluación de las intervenciones públicas en los que la transparencia es un valor predominante.

El concepto de producción de alianzas o «partenariado» público-privado, con respecto a la evaluación, abarcaría las diversas maneras de participación del sector privado con la financiación y explotación tanto de las propias evaluaciones como de la creación y desarrollo de las bases de datos que acumulen las distintas experiencias producidas. Debe entenderse desde una perspectiva amplia y globalizadora, en la que deben insertarse tanto la esfera gubernamental como los diversos actores sociales y grupos de interés en la evaluación, buscándose la producción de sinergias y complementariedad tanto en la mejora conceptual de las metodologías como en la implementación de bases de datos sobre los usos que de las evaluaciones se hayan podido formular.

Debe reconocerse la multiplicidad de centros de poder y de actores en la evaluación, lo que lleva implícito que la información y el conocimiento se mueven en múltiples direcciones y entre muchos y heterogéneos agentes y organizaciones sociales.

Para la consecución de un adecuado y deseable marco institucional, todos, evaluadores, expertos, técnicos y analistas, por un lado, y políticos y miembros de los poderes públicos, por otro lado, deben conciliar y converger en sus esfuerzos para institucionalizar la evaluación, para introducirla establemente en la gestión institucional, para dar a conocer objetivamente los indicadores precisos con los que medir problemas o demandas sociales y los resultados e impactos tras la puesta en práctica de unas o de otras políticas públicas, dotando a las evaluaciones del mayor rigor, cientificidad, objetividad, transparencia y credibilidad.

De ese modo, la sociedad en su conjunto y particularmente los actores sociales más involucrados en cada política, confiarán en la «institución» que pueda prestarles con calidad un buen servicio de evaluación, a partir del valor añadido que les supone acceder al conocimiento público y disponer de análisis rigurosos sobre los efec- 
tos que van teniendo las políticas que se han ido decidiendo o sobre el diagnóstico de un problema o sobre la panoplia de posibles decisiones a adoptar para solucionarlo.

De esta forma, la evaluación institucional transcurre hoy por espacios y ámbitos bien diferentes a los del pasado. Hay que asumir cierta responsabilidad de una evaluación «obsoleta» en la falta de usos eficientes de la misma por parte de decisores y gestores. Hoy existe una exigencia temporal y de oportunidad que debe tener respuesta en un incremento notable de la capacidad analítica-evaluadora para responder con celeridad a los nuevos retos. Sociedad del conocimiento, pluralidad de actores, incertidumbre, gestión de crisis, instan a la evaluación a ofrecer resultados operativos, disponibles en el espacio virtual, y, a la vez, rigurosos, para que puedan ser tenidos en cuenta por los decisores en los momentos oportunos.

La institucionalización de la evaluación no puede integrarse en paradigmas burocráticos incompatibles con las necesidades de una nueva gestión pública. Si es bien cierto que la evaluación es un instrumento clave para la gobernanza, no es menos cierto que solo una «nueva evaluación» puede aportar al buen gobierno. Construir estas nuevas formas de evaluación e integrarlas institucionalmente en los procesos de diseño e implementación de las políticas y programas públicos, son hoy, sin duda, retos nada desdeñables.

\section{Impulsar un Sistema Público de Evaluación: un compromiso de gobierno}

Al igual que sucede con otros países de la OCDE, en España ya existen instituciones que realizan evaluaciones, si bien de modo sectorial o más relacionadas con las políticas de gasto.

Por un lado, en la Administración del Estado se dispone de las evaluaciones de la programación, seguimiento y ejecución presupuestaria y control económico-financiero, que se realizan, como es conocido, por dos grandes instancias: el Tribunal de Cuentas, como supremo órgano fiscalizador de las cuentas y de la gestión económica del Estado y del sector público, y la Intervención General de la Administración del Estado $(\mathrm{IGAE})^{9}$ que, dentro del Ministerio de Economía y Hacienda, incorpora entre sus competencias las evaluaciones de la gestión y de las subvenciones ${ }^{10}$, y realiza auditorías para el análisis de la adecuación de los objetivos y de los sistemas de seguimiento y autoevaluación desarrollados por los órganos gestores, de la evaluación del resultado obtenido, de las alternativas consideradas y de los efectos producidos con relación a los recursos empleados.

${ }^{9}$ El artículo 72 de la Ley General Presupuestaria (Ley 47/2003, de 26 de noviembre) establece: «Evaluación de políticas de gasto. El Ministerio de Hacienda, en colaboración con los distintos centros gestores de gastos, impulsará y coordinará la evaluación continuada de las políticas de gasto con la finalidad de asegurar que las mismas alcancen sus objetivos estratégicos y el impacto socioeconómico que pretenden». La IGAE, según el artículo 140.2 de la Ley General Presupuestaria, ejerce «el control interno de la gestión económica y financiera del sector público estatal, con plena autonomía respecto de las autoridades y demás entidades cuya gestión controle». En la «exposición de motivos» de dicha Ley se establece que «las políticas de gasto pasan a ser el elemento nuclear de las asignaciones de los recursos, la evaluación de la gestión y el logro de los objetivos».

${ }^{10}$ En el caso de la Ley 38/2003, de 17 de noviembre, Ley General de Subvenciones, junto a la noción de evaluación de resultados, se hace referencia también a la evaluación como verificación ex-ante del cumplimiento de los requisitos de potenciales beneficiarios de subvenciones para decidir su concesión. El artículo 24.3 b) de dicha Ley de Subvenciones recoge la preevaluación y evaluación, como partes de la instrucción del procedimiento de concesión de subvenciones. Por su parte, la Exposición de motivos y el artículo 8 de la Ley hacen referencia a otro tipo de evaluación, ligada a la valoración y control de los planes estratégicos de subvenciones en los que se establecen las subvenciones. La disposición adicional 17. 'analmente atribuye a la IGAE la función de «control y evaluación de resultados derivados de la aplicación de los planes estratégicos...» 
Por otro lado, como es conocido, la evaluación de políticas y programas, así como de la calidad de determinados servicios públicos, está institucionalizada solo de manera sectorial en España, existiendo un marco legal para ello. Algunas de esas experiencias existentes han alcanzado un mayor grado de desarrollo, como las del sector de la enseñanza ${ }^{11}$ con la ANECA (Agencia Nacional de Evaluación de la Calidad y la Acreditación) o el INECSE (Instituto Nacional de Evaluación y Calidad del Sector Educativo); del sector sanitario, con la Agencia de Calidad del Sistema Nacional de Salud; del sistema público de ciencia y tecnología y de proyectos de I+D+i, con la ANEP (Agencia Nacional de Evaluación y Prospectiva); del sistema nacional del empleo; o del sector de la cooperación internacional al desarrollo, por citar algunos de los ejemplos más conocidos.

Junto a esas evaluaciones, también se desarrolla una labor menos conocida públicamente como es la de las Secretarías Generales Técnicas de los distintos Ministerios que, en relación con la calidad y el impacto normativo, colaboran en la evaluación ex-ante en el proceso de elaboración de leyes y reglamentos, en virtud de lo dispuesto en diversas normas de carácter estatal, existiendo funciones similares de evaluación normativa en algunas Comunidades Autónomas, algunas de las cuales, como la Generalitat de Cataluña ${ }^{12}$, obligan a incluir estudios económicos en términos de coste-beneficio.

Lo cierto es que existe una multiplicidad de disposiciones legales y órganos que, con objetivos diferentes y grados distintos de aplicación, prevén la posibilidad de la evaluación de políticas, aunque con ópticas departamentales o sectoriales, tanto en el seno de una propia Administración como de manera interdependiente entre distintos niveles de gobierno, tratándose en este último caso de políticas sobre todo presupuestadas con recursos provenientes de los fondos comunitarios europeos.

Desde una perspectiva sistémica y de visión de conjunto, con el análisis de las experiencias citadas se estarían evidenciando algunas carencias del modelo en su conjunto, al no estar precisamente articuladas las evaluaciones con otras experiencias o con algún marco común del que enriquecerse interadministrativamente. Los déficits se traducen en limitaciones a la extensión de la evaluación, al aprendizaje y usos de sus conclusiones, a la importancia dada a los resultados, al desconocimiento público generalizado o a la inexistencia de debates sobre los contenidos evaluativos.

Si a esas limitaciones le añadimos la necesidad, manifestada por los organismos internacionales y de la Unión Europea, de mejorar la regulación, utilizando para ello la evaluación ex-ante del impacto regulatorio, y tenemos en cuenta la transversalidad que caracteriza a muchas de las políticas y la corresponsabilidad financiera que sobre algunas se comparte entre los distintos niveles de gobierno, se inferiría que hoy es más evidente la necesidad de impulsar un sistema público de evaluación que dé un nuevo valor añadido a la sinergia de los esfuerzos en las distintas experiencias de evaluación ya existentes y a la articulación de las mismas.

${ }^{11}$ La evaluación se recoge de forma muy amplia en el Proyecto de Ley Orgánica de la Educación (LOE), actualmente en fase de discusión parlamentaria, extendiéndose a múltiples ámbitos: programación y organización (diseño), procesos y resultados de distinto tipo, centros, profesorado y función directiva. Puede establecerse una distinción entre una evaluación de carácter general, la evaluación del sistema educativo, y evaluaciones de carácter más específico: las generales de diagnóstico, de los centros y de la función directiva.

${ }^{12}$ La Ley 13/1989, de 14 diciembre, de Organización, Procedimiento y Régimen Jurídico de la Administración de la Generalidad de Cataluña (BOE de 12-01-1990 y DO Generalidad de Cataluña de 22-12-1989), modificada en distintas ocasiones por varias Leyes, señala en su artículo 63: «1. La elaboración de disposiciones de carácter general la inicia el centro directivo correspondiente. 2. La propuesta de disposición ha de ir acompañada de una memoria, la cual ha de expresar en primer lugar el marco normativo en el que la propuesta se inserta, ha de justificar su oportunidad y la adecuación de las medidas propuestas a los fines que se persiguen, ha de valorar la perspectiva de igualdad de género y ha de hacer referencia a las consultas que pueden haberse formulado y a otros datos de interés para conocer el proceso de elaboración de la norma. A la propuesta de disposición también se han de adjuntar: a) un estudio económico en términos de coste-beneficio; b) una lista de las disposiciones afectadas por la nueva propuesta; c) la tabla de vigencias de disposiciones anteriores sobre la misma materia, en la cual han de consignarse de forma expresa las que han de quedar total o parcialmente derogadas; d) un informe interdepartamental de impacto de género de las medidas establecidas en la disposición. 3. En los casos de creación de nuevos servicios o de modificación de los existentes, se adjuntará también un estudio del coste y financiamiento del mismo». 
La interdependencia entre organizaciones y Administraciones públicas evidencia también, de manera creciente, la necesidad de actitudes y de pautas legales de cumplimiento para la cooperación y colaboración entre ellas, a fin de asegurar unos mismos niveles de calidad entre las distintas organizaciones públicas y una mejora en el impacto de las políticas públicas del conjunto del Estado.

Con esas premisas, el actual gobierno español, en cumplimiento del programa que le supuso su victoria electoral, y a partir de la propia constitución del mismo, puso en marcha el proceso de creación de la Agencia Estatal de Evaluación de las Políticas Públicas y la Calidad de los Servicios.

La designación de una Comisión de Expertos de diferentes disciplinas académicas y profesionales constituyó uno de los primeros pasos para la creación de la citada Agencia ${ }^{13}$. La Comisión terminó sus trabajos en octubre de 2004, elevando el correspondiente Informe al Ministro de Administraciones Públicas.

En dicho Informe ${ }^{14}$, la propia Comisión reflejaba el carácter instrumental que tendría el objetivo de la Agencia y la manera más adecuada para alcanzarlo, partiendo de la convicción de que la evaluación ha de contar con un espacio diferenciado en el ámbito público y en el Estado descentralizado:

«La evaluación de políticas públicas constituye una actividad específica y con identidad propia, claramente distinta de otras como el control interno, la auditoría financiera, la auditoría de gestión o el control presupuestario, pero con los cuales mantiene y debe mantener una estrecha relación de complementariedad. Además de un espacio propio, la evaluación está llamada, también y sobre todo, a ocupar una posición central en el conjunto de organizaciones públicas. Al mismo tiempo, en un Estado políticamente descentralizado como el español, en el que el desarrollo de la acción pública requiere, en muchos casos, del concurso de los tres niveles de gobierno, también es preciso buscar un marco global de actuación en el que todos ellos tengan cabida y en el que exista una continua búsqueda del consenso en aspectos metodológicos y de funcionamiento» (p. 64) ${ }^{15}$.

\section{Como también se señalaba en el citado Informe, la Agencia debe:}

«[...] contribuir a la mejora de la acción y resultados en la actividad del sector público y sus instituciones, mediante la articulación de un Sistema Público de Evaluación de políticas públicas y de mejora de la calidad de los servicios, en un marco de transparencia y ética institucional» (p. 76).

La transferencia mutua interinstitucional de los conocimientos adquiridos y el favorecimiento del aprendizaje con las evaluaciones, sería uno de los objetivos finalistas de ese nuevo «sistema público de evaluación» que incorpore senderos donde transitar desde la deficitaria situación actual a nuevos escenarios donde institucionalizar la evaluación de políticas, a través del establecimiento de una Agencia Estatal.

${ }^{13}$ La Orden Ministerial APU/1463/2004, de 19 de mayo (BOE de 25 de mayo) designó la Comisión de Expertos encargada de «analizar la experiencia internacional, diagnosticar la situación de la evaluación en nuestro país y hacer las propuestas metodológicas e institucionales más deseables para la puesta en marcha de la Agencia».

${ }^{14}$ El Informe se hizo público con total inmediatez y fue divulgado en numerosos medios académicos y profesionales. Puede consultarse en la web oficial de la Dirección General de Inspección, Evaluación y Calidad de los Servicios del MAP 〈http://www.igsap.map.es/calidad/calidad.htm〉. Asimismo, y como ejemplo de la divulgación «externa», puede consultarse en la web de la Sociedad Española de Evaluación, en la sección «Recortes de Prensa, 25 de octubre de 2004: Presentación de los resultados del estudio realizado por la Comisión para el estudio y creación de la Agencia Estatal de Evaluación de la Calidad de los Servicios y de las Políticas Públicas en la sede de la UIMP en Valencia». 〈http://www.sociedadevaluacion.org/docs/informecomision.pdf). Asimismo, en 2005, el Informe de la Comisión de Expertos ha sido publicado por el INAP en forma de libro coordinado por mí, en el cual se recogen igualmente diversos trabajos complementarios que sirvieron de base para la elaboración de aquel Informe. [Esta obra es objeto de recensión, dentro de la sección correspondiente, en el presente número de la revista GAPP].

${ }^{15}$ Véase asimismo el trabajo del que el Informe extrae estas reflexiones: Vanaclocha, García Sánchez y Viñas (2005: 111-137). 
Dicha Agencia, que debiera gozar de independencia gubernamental, debe asumir sus roles con una estrategia de implantación de largo recorrido, para contribuir con sus funciones, valores y principios de funcionamiento, a favorecer la extensión de las prácticas de la evaluación y a coadyuvar a la mejora de los resultados de los procesos de implementación de las distintas políticas públicas.

Se trata de poder emitir un juicio de valor de carácter institucional, de forma organizada y científicamente contrastable, para que genere conocimiento, mejore la gestión pública, ayude a tomar mejores decisiones, y permita el aprendizaje del conjunto de las administraciones públicas y el debate plural y del conjunto de la ciudadanía y de los grupos en los que se organiza.

La creación de la Agencia Estatal de Evaluación de las Políticas Públicas y la Calidad de los Servicios, como elemento clave en el objetivo de institucionalización de la evaluación, fue autorizada por la disposición adicional primera de la Ley 28/2006, de 18 de julio, de Agencias Estatales para la mejora de los servicios públicos y, finalmente, su Estatuto ha sido aprobado por Real Decreto 1418/2006, de 1 de diciembre.

Antes de pasar a describir los elementos fundamentales de la citada Agencia, lo que será objeto del siguiente apartado, conviene resaltar aquí otros pasos importantes que se han dado en los últimos meses en la misma línea, en concreto, en el impulso de la calidad de los servicios y en la mejora del análisis del impacto regulatorio o normativo.

En efecto, la búsqueda del incremento de la calidad de los servicios públicos es precisamente la que orienta el compromiso gubernamental establecido con el marco general para la mejora de la calidad en la Administración General del Estado, aprobado ${ }^{16}$ por el Real Decreto 951/2005 de 29 de julio, que, junto a la creación de la Agencia, se convierte en otra pieza esencial de modernización de la Administración. Mejorar la calidad de los servicios públicos y fomentar la transparencia hacia los ciudadanos son los dos grandes objetivos del citado Real Decreto.

En dicha norma se concibe la calidad desde una visión que enmarca todas las pautas de la gerencia pública. Para mejorar estructural y establemente el funcionamiento de los servicios, la norma no pretende una yuxtaposición de iniciativas más o menos relacionadas, sino formar un marco en el que los programas recorren todo el ciclo de la gestión, desde la programación de objetivos a la ejecución de actividades, desde la simplificación y agilización de procedimientos a la evaluación de resultados, desde el liderazgo y las técnicas gerenciales a los desempeños de los empleados.

Con el nuevo marco contenido en el citado Real Decreto, el desarrollo de los programas de calidad se aproxima al centro de la gestión, subrayándose el imprescindible compromiso de los directivos para el éxito de los mismos. El Gobierno promueve que las organizaciones públicas definan sus objetivos de calidad en la prestación de servicios, a partir de la declaración explícita de sus compromisos ante la ciudadanía, que arbitren los mecanismos para cumplirlos, que sean transparentes y que permitan su evaluación continua, con la participación de los usuarios, para conocer sus resultados y poder rectificar y mejorar aquello que no funcione bien.

En coherencia con ese compromiso, se busca también la mejora efectiva de los resultados de los empleados en la prestación de servicios y el compromiso con los programas de calidad, a través de la mejora del complemento de productividad; se integran los programas de calidad con los procesos cotidianos de autoevaluación de las propias organizaciones públicas y con la evaluación externa de resultados a realizar por los órganos directivos competentes del departamento, asignándose a una unidad departamental las funciones de coordinación y seguimiento global de los programas, y atribuyendo a la correspondiente Inspección General de Servicios la realización de evaluaciones de la calidad.

${ }^{16}$ Real Decreto 951/2005, de 29 de julio (BOE de 3 de septiembre), por el que se establece el marco general para la mejora de la calidad en la Administración General del Estado. 
Con la nueva norma se enfatiza la participación de los usuarios y ciudadanos a través de diversos programas. Con el programa de «Quejas y Sugerencias» se conciben a éstas como una herramienta más de detección de la voz del usuario y de mejora continua de los servicios de atención e información. Con el innovador programa de «Análisis de la demanda y de evaluación de la satisfacción de los usuarios» se regulan por primera vez de forma explícita las prácticas de consulta a los usuarios de los servicios.

Cabe resaltar en este artículo que, con el programa del «Observatorio de la calidad», se recupera un proyecto pionero de los años 1993-1995, que en su momento abrió el camino para las iniciativas de calidad en nuestra Administración, y así fue reconocido por el Comité P.U.M.A. de la OCDE. Se trata ahora de poner en funcionamiento una plataforma que haga posible el análisis periódico y regular de la percepción social sobre los servicios públicos, contando con informadores representativos de los agentes socioeconómicos, con la finalidad de proponer iniciativas generales de mejora y facilitar a la ciudadanía, junto a la evaluación que se realice, información global sobre la calidad de los servicios prestados por la Administración pública.

Finalmente, el Real Decreto introduce algunas modificaciones a las normas anteriores de Calidad en la Administración General del Estado, a partir de las enseñanzas extraídas de las experiencias desarrolladas en los últimos años. De ese modo, el programa de «Premios» transciende el ámbito exclusivo de la Administración General del Estado y se convierte en un programa de ámbito estatal y para el conjunto de las Administraciones públicas. Los «Premios a la Calidad e Innovación en la Gestión Pública» estarán abiertos a candidaturas de los tres niveles de gobierno (central, autonómico y local). Además, junto con los premios, se crea el procedimiento de «reconocimiento», en virtud del cual las organizaciones que hayan obtenido los mejores resultados conforme a los modelos de calidad y excelencia obtendrán incentivos al desempeño.

El segundo ámbito al que se ha hecho referencia es el de la mejora del análisis del impacto regulatorio o normativo. En este caso, si bien no ha sido todavía aprobada la norma correspondiente, existe un mandato aprobado por el Consejo de Ministros en el pasado mes de febrero ${ }^{17}$ para desarrollar la Ley del Gobierno, al objeto de sistematizar el análisis del impacto regulatorio que debe acompañar a los proyectos normativos, esto es, lo que se ha venido denominando la «evaluación ex-ante». Sin duda, el desarrollo de este mandato supondrá un paso más en el avance hacia la institucionalización de la práctica y la cultura de la evaluación.

\section{El contenido y características de la Agencia de Evaluación y Calidad}

En el Estatuto de la Agencia Estatal de Evaluación de las Políticas Públicas y la Calidad de los Servicios (en adelante Agencia de Evaluación y Calidad) se establece que los objetivos de la Agencia son:

a) Promover la cultura de evaluación y de calidad de los servicios e impulsar su práctica en la gestión pública.

b) Elaborar y proponer metodologías, realizar actividades de acreditación y certificación, en los términos previstos en el presente Estatuto, y fomentar la implantación de sistemas de información e indicadores, para la evaluación y la gestión de la calidad.

c) Realizar trabajos de evaluación y análisis de políticas y programas públicos.

d) Fomentar la mejora de la calidad de los servicios públicos como compromiso con la ciudadanía.

${ }^{17}$ Resolución de 1 de abril de 2005, de la Subsecretaría del Ministerio de Presidencia, por la que se dispone la publicación del Acuerdo del Consejo de Ministros, de 25 de febrero, por el que se adoptan mandatos para poner en marcha medidas de impulso a la productividad (BOE de 2 de abril). 
e) Analizar, a efectos de la realización del Informe previsto en el apartado 3 de la disposición adicional primera de la Ley de Agencias Estatales, la actividad desplegada por las agencias estatales, y su compromiso para mejorar la calidad de los servicios prestados a los ciudadanos.

f) Prestar un servicio eficaz, eficiente y de calidad, en un marco donde se equilibren la responsabilidad por la gestión y la autonomía y flexibilidad de la misma.

Para la consecución de los anteriores objetivos, la Agencia de Evaluación y Calidad ejercerá las siguientes competencias y funciones más relevantes:

- Impulsar y fomentar la cultura de la evaluación de la gestión de la calidad a través del desarrollo e impulso de actividades de formación, investigación y publicación, así como del intercambio de experiencias y mejores prácticas.

- Proponer el establecimiento y armonización de indicadores, sistemas de información, metodologías y guías de evaluación de las políticas y servicios públicos, actuando como entidad de referencia para el diseño de los sistemas de acreditación y certificación, así como de certificación de organizaciones y acreditación de certificadoras de productos y procesos tales como «Cartas de Servicios» y «Buenas Prácticas».

- Ejecutar trabajos de evaluación y análisis de políticas públicas.

- Prestar servicios de asistencia técnica, consultoría y análisis de evaluaciones realizada por terceros a solicitud de los órganos gestores e instituciones.

- Preparar la propuesta de planes de evaluación de políticas y programas públicos para su aprobación por el Gobierno.

Y tales funciones, en sintonía con la propuesta del informe de la Comisión de Expertos y con los criterios construidos a partir de las experiencias acumuladas por otras organizaciones de ámbito internacional y de las propias organizaciones sectoriales, debieran desempeñarse por la Agencia Estatal de Evaluación a partir de diversos principios y características inspiradoras de funcionamiento, que conforman el proyecto gubernamental:

- Siendo capaz de generar credibilidad institucional y social, por su independencia de criterio, responsabilidad y profesionalidad.

- Trabajando con una estructura reducida en número de personas, pudiendo incorporar, en programas y proyectos específicos, a los mejores expertos académicos, analistas y gestores nacionales e internacionales en políticas públicas.

- Integrando, en sus miembros, la pluralidad profesional y metodológica subyacente a los procesos de evaluación, y siendo flexible para adaptarse a nuevas necesidades y a la externalización de sus tareas complementarias.

- Siendo experta en procesos de evaluación y habilidades gerenciales que implican la dirección de evaluaciones de políticas transversales, europeas, en curso o de nueva formulación, y aquellas de marcado impacto social y económico.

- Centrándose en los procesos institucionales de naturaleza formativa de investigación, cultural y metodológica.

- Configurando su acción en torno a la interdependencia, como organización de nuevo tipo capaz de generar la colaboración más amplia entre el conjunto de actores públicos y privados que conforman el proceso-red de evaluación de las políticas públicas. 
- Impulsando el desarrollo del Sistema Público de Evaluación y abriéndose a la participación y colaboración con las Comunidades Autónomas y otras Administraciones Territoriales;

- Procurando que gradualmente pueda constituirse en un referente de prestigio nacional e internacional.

- Siendo susceptible de cambio y evolución hacia nuevos diseños institucionales en el futuro.

La constitución de la Agencia de Evaluación y Calidad se inscribe en el nuevo modelo gestor establecido por la Ley de Agencias Estatales, sustentando en los principios de autonomía, de responsabilidad y de control que son instrumentalizados a través del Contrato de gestión en los términos fijados en el Estatuto de la Agencia

El contrato de gestión tiene por objeto regular la actividad de la Agencia y las relaciones recíprocas entre la misma y los Ministerios de Administraciones Públicas y de Economía y Hacienda, al estar adscrita al primero y en cuanto a la financiación de su actividad al segundo.

El Consejo de Ministros de 30 de marzo de 2007 adoptó el Acuerdo por el que se aprueban los programas y políticas públicas que serán objeto de evaluación por la Agencia en 2007, la cual deberá incluirlos en su Plan de Trabajo anual.

La innovación legislativa con la que institucionalizar la evaluación de políticas en nuestro país debe percibirse como un valor añadido de un nuevo servicio puesto a disposición de partidos e instituciones, de la ciudadanía y de los múltiples actores sociales involucrados, siendo, además, una de las finalidades esenciales de la creación de la Agencia Estatal de Evaluación ayudar a la mejora de la calidad democrática de la sociedad española.

Una Agencia con las características de comportamiento antes señaladas, debe ganarse su credibilidad y confianza a partir de unos desempeños que nítidamente favorezcan la incorporación de los sistemas de evaluación a los procesos decisorios, a fin de mejorar, en definitiva, los resultados de las políticas públicas de nuestro país para satisfacción de la sociedad española.

\section{REFERENCIAS BIBLIOGRÁFICAS Y DOCUMENTALES}

Conseil Scientifique de l'Evaluation (1996), Petit Guide de l'évaluation des politiques publiques. París: La Documentation française $\langle$ http://www.evaluation.gouv.fr/cgp/fr/interministere/doc/petit_guide_cse.pdf).

Derlien, H.U. (1998), «Una comparación internacional en la evaluación de las políticas públicas», Gestión y Análisis de Políticas Públicas 11-12: 14-15.

Ferrera, M. (1991), «El análisis de políticas públicas en Italia», Documentación Administrativa 224-225: 195-210.

Garde, J.A., coord. (2005), La Agencia Estatal de Evaluación de la calidad de los Servicios y de las Políticas Públicas: Reflexiones y propuesta de creación. Informe Comisión Expertos. Madrid: INAP.

Monnier, E. (1995), Evaluación de la acción de los poderes públicos. Madrid: IEF-MEH.

NN.UU. Grupo de Evaluación de las Naciones Unidas, UNEG (2005), Normas de Evaluación en el sistema de Naciones Unidas 〈http://www.unicef.org/spanish/evaluation/index_4715.html〉.

Perret, B. (2001), L'evaluation des politiques publiques. París: La Decouverte. 
Rivera, M. y Fernández y Zavala, L., comp., (2002), Evaluación programática y educacional en el sector público. Enfoque y perspectivas («Primera parte»: Evaluación y Estado en América Latina). Washington D.C.: Agencia Interamericana para la Cooperación y el Desarrollo. 〈http://www.iacd.oas.org/template-spanish/educ1-s.htm〉.

Subirats, J. (1989), Análisis de políticas públicas y eficacia de la Administración. Madrid: INAP.

Vanaclocha, FJ., García Sánchez, E., y Viñas, V. (2005), «Algunas referencias sobre metodología de la evaluación de las políticas y los servicios públicos», en J.A. Garde, coord., La Agencia Estatal de Evaluación de la calidad de los Servicios y de las Politicas Públicas: Reflexiones y propuesta de creación. Informe Comisión Expertos. Madrid: INAP.

Viñas, V. (2001), «El uso de técnicas cualitativas en la evaluación de programas. Los programas de desarrollo regional financiados por la Unión Europea», Revista Española de Investigaciones Sociológicas 9: 155-177.

Viñas, V. (2002), «Descripción y análisis de la teoría sobre seguimiento y evaluación aplicable a las actuaciones cofinanciadas por Fondos Europeos», Gestión y Análisis de Politicas Públicas 25: 3-21.

Zapico, E. (2005), «Comisiones de análisis de programas y asignación eficiente del gasto público», Revista Economistas 105: 43-44. 